\title{
Elevada incidência de anormalidades cromossômicas numéricas detectadas por FISH multicentromérico em pacientes com mieloma múltiplo
}

High incidence of chromosomal numerical abnormalities by multicentromeric FISH in multiple myeloma patients

Maria de Lourdes L. F. Chauffaille'; Aníbal Ribeiro Jr. ${ }^{2}$; Mihoko Yamamoto ${ }^{3}$; Maria Madalena Rodrigues ${ }^{4}$; Manuella S. S. Almeida ${ }^{2}$; Christian Ribas ${ }^{2}$; Luis A. Calheiros ${ }^{2}$; Giselle W. B. Colleoni ${ }^{3}$

abstract

This study aimed to characterize genetic alterations by interphase multicentromeric FISH focusing on chromosomal numerical abnormalities and using some locus specific probes for the most frequent aberrations found in the disease, in a homogeneous cohort of 34 advanced stage, but recently diagnosed MM patients; $97 \%$ had numerical chromosomal abnormalities detected by FISH, being 75\% hyperdiploid, 18\% hypodiploid and $3 \%$ tri/tetraploid. Using locus specific probes, we found 13 q deletion in $30 \%$ and IGH rearrangement in $25 \%$ of cases. Grouping hypodiploid patients together with del13q (unfavorable group) and comparing them to the remaining cases (non unfavorable group) we found a trend towards younger patients presenting more unfavorable abnormalities $(p=0.06)$ and significant lower hemoglobin level $(\mathrm{Hb}<8.5 \mathrm{mg} / \mathrm{dl}, p=0.03)$.

\footnotetext{
\begin{tabular}{|c|c|}
\hline unitermos & resumo \\
\hline Mieloma múltiplo & Este estudo objetivou detectar as alterações genéticas em pacientes com mieloma múltiplo (MM), usando \\
\hline Cariótipo & o método de hibridação in situ por fluorescência em interfases (FISH interfásico). Para detectar as alterações \\
\hline & numéricas foram usadas sondas multicentroméricas e para os rearranjos mais freqüentemente observados \\
\hline Aneuploidia & na doença foram utilizadas as sondas lócus específicas para IGH, P53, ciclina D1 e RB1. Foram estudados 34 \\
\hline FISH & pacientes com MM em estágio avançado, ainda que recém-diagnosticados, $97 \%$ dos quais apresentaram \\
\hline & anormalidades numéricas detectadas por FISH, sendo $75 \%$ hiperdiplóides, $18 \%$ hipodiplóides e $3 \%$ tri/tetra- \\
\hline & plóides. Em relação às demais anormalidades, a deleção $13 q$ foi encontrada em $30 \%$ dos casos e o rearranjo \\
\hline & $\begin{array}{l}\text { IGH, em } 25 \% \text {. Agrupando os pacientes com hipodiploidia e com deleção 13q14 (grupo desfavorável) e } \\
\text { comparando-os com os demais (grupo não-desfavorável), houve tendência a pacientes jovens no grupo des- } \\
\text { favorável }(p=0,06) \text { e níveis de hemoglobina }(\mathrm{Hb}) \text { significativamente mais baixos }(<8,5 \mathrm{~g} / \mathrm{dl}, p=0,03 \text { ). }\end{array}$ \\
\hline
\end{tabular}

unitermos

Aneuploidia

favorável $(p=0,06)$ e níveis de hemoglobina $(\mathrm{Hb})$ significativamente mais baixos $(<8,5 \mathrm{~g} / \mathrm{dl}, p=0,03)$.
}

\section{Introdução}

O mieloma múltiplo (MM) é neoplasia clonal de célula $ß$, que se caracteriza pelo acúmulo de plasmócitos produtores de imunoglobulina. A sobrevida dos pacientes acometidos por MM varia de alguns meses a vários anos, o que traduz a grande heterogeneidade da doença ${ }^{(8)}$. O MM é atualmente considerado uma doença incurável, apesar dos avanços no tratamento, entre os quais se destaca a alta dose de quimioterapia seguida por transplante autólogo de células-tronco hematopoéticas ${ }^{(11)}$. Na verdade, um dos desafios para o hematologista é estabelecer a melhor opção terapêutica que possibilite uma remissão prolongada. Nesse sentido,

1. Professora-associada da disciplina de Hematologia e Hemoterapia da Universidade Federal de São Paulo (UNIFESP).

2. Pós-graduandos da disciplina de Hematologia.

3. Professores-adjuntos da disciplina de Hematologia

4. Bióloga.

Projeto financiado pela Fundação de Amparo à Pesquisa do Estado de São Paulo (Fapesp) nº 01/13086-3 
a identificação de características clinicolaboratoriais que afetem a resposta ao tratamento e a sobrevida dos pacientes com MM é essencial para o planejamento terapêutico.

Embora a detecção de aberrações genéticas em células de MM tenha aumentado o entendimento da patogênese da doença ${ }^{(1)}$, as implicações clínicas e prognósticas dessas alterações heterogêneas ainda não foram completamente elucidadas.

Os estudos cromossômicos em MM são, até certo ponto, limitados devido à dificuldade na obtenção de metáfases, já que os plasmócitos malignos apresentam, via de regra, baixo índice mitótico ${ }^{(14)}$. A análise do cariótipo por banda G permite a detecção de $20 \%$ a $50 \%$ de casos anormais ${ }^{(4,}$ 14). Pelo método da hibridação in situ por fluorescência (FISH), os diferentes cromossomos podem ser enumerados e é possível detectar alta incidência de anormalidades numéricas com o uso de sondas multicentroméricas ${ }^{(4)}$. Porém, o cariótipo convencional ainda guarda seu valor, ao diagnóstico, por auxiliar na identificação de alterações com significado prognóstico.

O objetivo deste estudo foi detectar alterações cromossômicas numéricas (com auxílio de sonda multicentromérica), e alterações estruturais, como a translocação t $(11 ; 14)$, deleção $13 q 14$, correspondente ao gene RB1, deleção 17p13.1, correspondente ao gene P53, e rearranjos envolvendo IGH (14q32.3) por FISH interfásico, em amostras de medula óssea de pacientes com diagnóstico de MM provenientes de hospital público, para correlacioná-las com alguns aspectos clinicolaboratoriais.

\section{Casuística e método}

Foram estudados 34 casos de MM, diagnosticados entre maio de 2002 e janeiro de 2004, na Universidade Federal de São Paulo/Escola Paulista de Medicina (Unifesp/EPM) e no Hospital do Servidor Público Estadual Francisco Morato de Oliveira (HSPE-FMO), após consentimento informado. Dezoito pacientes eram do sexo masculino e 16 do feminino (relação de 1,8:1), com mediana de idade de 60 anos (variando de 40 a 79 anos). De acordo com o sistema de estadiamento de Durie e Salmon ${ }^{(5)}, 32$ (94\%) pacientes foram diagnosticados em estádio III e apenas dois em estádio I da doença.

A $\beta 2$ microglobulina ( $\beta 2 \mathrm{MG}$ ) foi maior que $3,5 \mathrm{mg} / \mathrm{dl}$ em $74 \%$ dos casos e a albumina foi inferior a 3,5 g/dl em $60 \%$ dos casos. Segundo o sistema internacional de escore prognóstico (ISS) ${ }^{(7,9)}, 3$ (10\%) dos pacientes foram classifi- cados como escore I; 13 (42\%) como II e 15 (48\%) como III, isto é, 90\% apresentaram escores ISS elevados. Sessenta e quatro por cento dos casos apresentaram isotipo lgG e 74\% secretavam cadeia leve kappa.

O FISH foi realizado nas células mononucleares da medula óssea com as seguintes sondas: Chromoprobe Multiprobe-I system (Cytocell, UK) para enumeração centromérica, LSI cyclin D1 spectrum orange/CEP 11 spectrum green; (11q13) (Vysis Inc., USA.); LSI 13/RB1 DNA spectrum orange (13q14) (Vysis Inc., USA); LSI p53 spectrum orange (17p13.1) (Vysis Inc., USA) e LSI IGH dual color break a part probe (14q32.3) (Vysis Inc., USA), de acordo com as instruções do fabricante. As células foram analisadas em microscópio de fluorescência (Axioskop 2 Plus - Carl Zeiss Corporation, Germany). Os sinais de hibridação foram avaliados em 100 células/observador, por dois observadores. As imagens foram digitalizadas em computador com o programa MacProbe 4.4 para FISH da Power Gene System (Applied Imaging Corporation, USA).

Para controle interno da reação de hibridação foram usadas cinco amostras de medula óssea de doadores voluntários de medula para transplante, após consentimento informado. Essas amostras serviram tanto de controle intra-reação como para determinar o nível de hibridação para células, com um ou mais domínios para cada sonda em células hematopoéticas normais. Os níveis de corte para o diagnóstico de aneuploidia foram definidos pela média mais 2 desvios-padrão dos sinais observados nas amostrascontrole. As associações entre as variáveis dicotômicas de interesse foram testadas com o teste qui-quadrado $\left(\mathrm{X}^{2}\right)$ de Pearson. Este estudo foi aprovado pelo Comitê de Ética em Pesquisa da UNIFESP.

\section{Resultados}

Noventa e sete por cento dos casos, ou seja, todos exceto um (caso 9) apresentaram alteração cromossômica numérica detectada pelo FISH interfásico (Tabela 1). Entre esses casos aneuplóides, 25 (75\%) eram hiperdiplóides; 6 (18\%) hipodiplóides; 1 (3\%) triplóide/tetraplóide (caso 28) e outro era pseudodiplóide (caso 34). Com uso de sondas lócus específicas, a deleção 13q (del13q) foi observada em 10 de 33 casos (30\%) e rearranjo IGH em 8 de 32 casos (25\%). Não se observaram deleção p53 e/ou rearranjo envolvendo ciclina D1 na presente casuística (Tabela 2). Devido à escassez de amostra, o caso 4 foi testado apenas para a sonda multicentromérica e os casos 24 e 32 não foram testados para ciclina D1 e rearranjo $\mathrm{IGH}$, respec- 
tivamente. Três casos (5, 20 e 28) (30\%), entre os nove não-hiperdiplóides, apresentaram deleção $13 q$ simultânea às alterações numéricas e dois deles (casos 5 e 28) apresentaram evolução desfavorável, alcançando óbito por doença agressiva em curto período de tempo.

Embora seja realizado de forma rotineira, nem todos os casos apresentaram resultado de cariótipo pelo método convencional por banda $G$, porém entre aqueles com resultado disponível, $21 \%$ apresentaram alterações (casos $15,17,19,23$ e 28$)$.

Ao agrupar os pacientes com hipodiploidia com aqueles com deleção 13q (hipo/del13q) (grupo de prognóstico desfavorável) ${ }^{(13)}$ e comparar com os casos remanescentes, em relação à idade, concentração de hemoglobina $(\mathrm{Hb})$, $\beta 2 \mathrm{MG}$, creatinina, cálcio sérico e desidrogenase lática (DHL) (Tabela 3), dados interessantes puderam ser extraídos. Com relação à idade, 9 de 27 pacientes (33\%) com mais de 50 anos apresentaram anomalias cromossômicas desfavoráveis versus a maioria $(5 / 7,71 \%)$ daqueles com menos de 50 anos, percebendo-se então uma tendência para pacientes jovens apresentarem mais anomalias desfavoráveis $(p=0,06)$. No tocante à concentração de $\mathrm{Hb}$, $64 \%$ do grupo hipo/del13q apresentou $\mathrm{Hb}<8,5 \mathrm{mg} / \mathrm{dl}$ versus menos de $30 \%$ dos demais $(p=0,03)$. Em relação ao cálcio, todos os casos favoráveis apresentaram cálcio < $11,5 \mathrm{mg} / \mathrm{dl}$ contra $72,7 \%$ dos do grupo desfavorável ( $p=$ $0,09)$. Não houve diferença em relação à $\beta 2 \mathrm{MG}, \mathrm{DHL}$ e creatinina entre ambos os grupos.

\section{Tabela 1 Resultado do FISH multicentromérico}

\begin{tabular}{|c|c|c|}
\hline & Cariótipo & Sistema Multiprobe I \\
\hline 1 & $46, \mathrm{XY}[8]$ & $+3(4),+9(6),-13 / 21(35)$ \\
\hline 2 & $46, \mathrm{XX}[7]$ & ${ }^{*}+1(18),+7(0,5),+9(29),-13 / 21(34), 14 / 22(26,5)$ \\
\hline 3 & $46, \mathrm{XX}[5]$ & $\begin{array}{l}-1(15,5),+1(15),+2(13,5),+2+2(32,5),+3(64,5),+6(34),+7 \\
(60,5),+9(33),+10(2,5),-13 / 21(35),-13 / 21-13 / 21(32),-14 / 22 \\
(65),+15(22),+16(0,5),+17(38),+18(28),+20(0,5),-X(42)\end{array}$ \\
\hline 4 & $46, \mathrm{XX}[2]$ & $-6(20),+6(25),-7(21),-8(11,5),-11(15,5),-13 / 21(32,5),+15(6)$ \\
\hline 5 & Sem metáfases & $\begin{array}{l}*+1(9),+3(19),-7(21),+9(20),-11(18,5),-13 / 21(30,5),-15 \\
(26),-18(15)\end{array}$ \\
\hline 6 & Sem metáfases & $-1(16),+9(18,5),+11(10),-17(13,5),-18(16,5)$ \\
\hline 7 & $46, \mathrm{XY}[2]$ & $\begin{array}{l}+4(35),+6(10,5),+7(4,5),+9(14),-12(5,5),-13 / 21(84,5), \\
-14 / 22(35),+15(9,5),+18(11),-X(21)\end{array}$ \\
\hline 8 & Sem metáfases & $+2(8,5),-4(1,5),-13 / 21(44),-15(15,5)$ \\
\hline 9 & Sem metáfases & Normal by FISH \\
\hline 10 & $46, \mathrm{XY}[2]$ & $+7(2),+9(1),+10(3),+12(10)$ \\
\hline 11 & $46, \mathrm{XX}[6]$ & $+1(9),-7(9),-12(5),-13 / 21(28),-15(21),-16(14,5)$ \\
\hline 12 & $46, \mathrm{XX}[4]$ & $+6(9),+7(5),+9(2),+15(3),-X(10)$ \\
\hline 13 & $46, \mathrm{XX}[7]$ & +9 (37), +9 +9 (35), +12(2), +17 (2,5), -X (15) \\
\hline 14 & Sem metáfases & $\begin{array}{l}+1(25),+6(1,5),+7(3),+9(6,5),+10(4,5),+10+10(1,5),+12+12(4,5), \\
-13 / 21(35),-14 / 22(38,5),+15(31,5),+16(1),+17(1),+20(1)\end{array}$ \\
\hline 15 & $45, X Y,-9[2]$ & $\begin{array}{l}+7(12),-9(15,5),+11(6,5),+12(3),+15(5),+17(2,5),-18(13) \\
+18(7,5)\end{array}$ \\
\hline 16 & $46, X Y[2]$ & $+3(10),+6(4),+7(4),-15(20)$ \\
\hline 17 & $43, X,-Y,-5,-17[1]$ & $\begin{array}{l}+1(17),+3(10),+6(2),-9(29),+11(2),-13 / 21(56),-15(15),+17 \\
(1,5),-Y(37,5)\end{array}$ \\
\hline 18 & Sem metáfases & $+6(3),+7(3),-13 / 21(27)$ \\
\hline 19 & $46, X Y[10] / 45 \sim 46, X Y,-1,+3,-4,-13[3]$ & $+3(26),+7(3,5),+17(30),-13 / 21(34)$ \\
\hline 20 & $46, X Y[12]$ & $+7(1),+9(2),-11(10),-12(5),-13 / 21(28),-17(11)$ \\
\hline
\end{tabular}




\begin{tabular}{lll}
21 & $46, X Y[3]$ & $+7(3)$ \\
22 & $46, X X[8]$ & $+7(5),+8(2),+9(12),+11(1),-12(10),+12(3),+15(7),+16(1)$, \\
& $-17(12),+17(20)$ \\
23 & $46, X Y[6] / 47, X Y,+17[2]$ & $+17(1)$ \\
24 & $46, X Y[8]$ & $+3(4),+7(12),+15(2,5)$ \\
25 & $46, X Y[3]$ & $-9(20)$ \\
26 & $46, X Y[6]$ & $+6(3),+7(2),+9(4),+10(2),+16(4),+17(5),+20(5)$ \\
27 & Sem metáfases & $-7(7),+7(1),+15(7),+16(1)$ \\
28 & $46, X Y[3] / 78 \sim 81, X X X Y Y[7]$ & $+1(12),+3+3(8),+6(9),+6+6(1),+7(3),+7+7(3),+8(2),+9$ \\
& & $(5),+11(4),+12(3),+12+12(2),-13 / 21(64),-15(17),+15(9)$, \\
& $+16(2,5),+16+16(1,5),+17(2)$ \\
29 & $46, X X[3]$ & $+8(2),+9(1),+11(1)$ \\
30 & $46, X Y,[9]$ & $+4(4),+9(1),-13 / 21(32),+17(1)$ \\
31 & $46, X X[2]$ & $-8(28),+9(4,5),+15(4)$ \\
32 & Sem metáfases & $*$ \\
33 & Sem metáfases & $* 3(18)$ \\
34 & Sem metáfases & $* 8(6),+9(3),+15(5),+16(4),+17(4)$ \\
\hline
\end{tabular}

Legenda: nos [ ] e nos () estão especificados o número de metáfases e interfases encontradas com aquela alteração cromossômica numérica, respectivamente

Tabela 2 Anormalidades encontradas pelas diferentes sondas lócus específicas

\begin{tabular}{|c|c|c|c|c|c|}
\hline Caso & Multicentromérica & Cromossomo $13 q 14$ & IgH & p53 & Ciclina D1 \\
\hline 1 & Hiper & Normal & Normal & Normal & DEL \\
\hline 2 & Hiper & Normal & Normal & Normal & Normal \\
\hline 3 & Hiper & Normal & Normal & Normal & Normal \\
\hline 4 & Hipo & $\mathrm{NR}$ & NR & NR & $\mathrm{NR}$ \\
\hline 5 & Hipo & Del & Normal & Normal & Mono \\
\hline 6 & Hiper & Normal & Rearranjo & Normal & Normal \\
\hline 7 & Hiper & Del & Normal & Normal & Normal \\
\hline 8 & Hipo & Normal & Normal & Normal & Normal \\
\hline 9 & Normal & Normal & Normal & Normal & Normal \\
\hline 10 & Hiper & Normal & Normal & Normal & DEL \\
\hline 11 & Hipo & Normal & Normal & Normal & Desconhecida \\
\hline 12 & Hiper & Normal & Monossomia & Normal & Normal \\
\hline 13 & Hiper & Normal & Monossomia & Normal & Normal \\
\hline 14 & Hiper & Del & Normal & Normal & Normal \\
\hline 15 & Hiper & Normal & Normal & Normal & Normal \\
\hline 16 & Hiper & Normal & Normal & Normal & Normal \\
\hline 17 & Hiper & Del & Rear/mono/del & Normal & Normal \\
\hline 18 & Hiper & Normal & Normal & Normal & Normal \\
\hline 19 & Hiper & Del & Normal & Normal & Normal \\
\hline 20 & Hipo & Del & Normal & Normal & Normal \\
\hline
\end{tabular}




\begin{tabular}{lccccc}
\hline 21 & Hiper & Normal & Normal & Normal & Normal \\
22 & Hiper & Normal & Normal & Normal & Normal \\
23 & Hiper & Normal & Normal & Normal & Normal \\
24 & Hiper & Normal & Rearranjo & Normal & NR \\
25 & Hipo & Normal & Normal & Normal & Normal \\
26 & Hiper & Del & Normal & Normal & Normal \\
27 & Hiper & Del & Rearranjo & Normal & Normal \\
28 & Tri/Tetra & Del & Rearranjo & Normal & Tetra/mo \\
29 & Hiper & Del & Rear./mono & Normal & Normal \\
30 & Hiper & Normal & Normal & Normal & Normal \\
31 & Hiper & Normal & Rearranjo & Normal & Normal \\
32 & Hiper & Normal & NR & Normal & Trissomia \\
33 & Hiper & Normal & Normal & Normal & Desconhecida \\
34 & Pseudodiplóide & Normal & Rearranjo & Normal & Normal \\
\hline
\end{tabular}

Hiper = hiperdiplóide; Hipo = hipodiploide; NR = não realizado; Del = deleção; NA = não avaliado; RP = resposta parcial; SR = sem resposta; mono: monossomia.

\begin{tabular}{|c|c|c|c|c|}
\hline \multirow[t]{2}{*}{ Tabela 3} & \multicolumn{4}{|c|}{$\begin{array}{l}\text { Pacientes hipodiplóide/del13q versus os demais em relação a determinados aspectos } \\
\text { clinicolaboratoriais }\end{array}$} \\
\hline & Hipodiplóide & e/ou de13q14 & Demais pacientes & $p$ \\
\hline \multirow[t]{2}{*}{ Idade (anos) } & $<50(7)$ & 5 & 2 & \\
\hline & $\geq 50$ & 9 & 18 & 0,06 \\
\hline \multirow[t]{2}{*}{$\mathrm{Hb}(\mathrm{g} / \mathrm{dl})$} & $<8,5(15)$ & 9 & 6 & \\
\hline & $\geq 8,5$ (19) & 5 & 14 & 0,03 \\
\hline \multirow[t]{2}{*}{$\beta 2 \mathrm{M}(\mathrm{mg} / \mathrm{l})$} & $<3,5(8)$ & 3 & 5 & \\
\hline & $\geq 3,5(23)$ & 10 & 13 & 0,76 \\
\hline \multirow[t]{2}{*}{ Albumina ( $\mathrm{g} / \mathrm{dl})$} & $<3,5(20)$ & 9 & 11 & \\
\hline & $\geq 3,5$ (13) & 4 & 9 & 0,41 \\
\hline \multirow[t]{2}{*}{ Creatinina (mg/dl) } & $<2(21)$ & 7 & 14 & \\
\hline & $\geq 2(13)$ & 7 & 6 & 0,29 \\
\hline \multirow[t]{2}{*}{ Calcio (mg/dl) } & $<11,5(20)$ & 8 & 12 & \\
\hline & $\geq 11,5(3)$ & 3 & 0 & 0,09 \\
\hline \multirow[t]{2}{*}{ DHL (U/L) } & $<240(7)$ & 3 & 4 & \\
\hline & $\geq 240$ (21) & 10 & 11 & 0,43 \\
\hline \multirow[t]{3}{*}{ Isótipo } & Light chain (6) & 4 & 2 & \\
\hline & $\operatorname{IgG}(22)$ & 8 & 14 & 0,37 \\
\hline & $\operatorname{IgA}(6)$ & 2 & 4 & \\
\hline \multirow[t]{2}{*}{ Cadeia leve } & Kappa (19) & 6 & 13 & \\
\hline & Lambda (12) & 7 & 5 & 0,14 \\
\hline \multirow[t]{3}{*}{ ISS } & I (3) & 1 & 2 & \\
\hline & II (13) & 6 & 7 & \\
\hline & III (15) & 6 & 9 & 0,90 \\
\hline
\end{tabular}

DHL: desidrogenase láctica. 


\section{Discussão}

O aparecimento das alterações cromossômicas no MM está relacionado aos rearranjos gênicos, que provocam a produção das proteínas responsáveis pela proliferação celular e pelo bloqueio da apoptose dos plasmócitos. A literatura apresenta o envolvimento das translocações (13q14 com o gene RB1; 17p13 com p53; 14q32 com IGH; e 11 q13 com ciclina D1), demonstrando a importância de se conhecer as alterações cromossômicas e suas relações com o gene e/ou as proteínas anormais que aparecem na

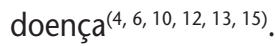

Neste estudo foram caracterizadas as alterações genéticas por FISH multicentromérico, com foco nas alterações numéricas, e utilizando-se sondas lócus específicas para as aberrações mais freqüentemente encontradas na doença, em um grupo de pacientes com MM em estágio avançado, ainda que recém-diagnosticados.

O cariótipo por banda G apresenta habitualmente elevada taxa de resultado normal em MM devido ao baixo índice mitótico dessas células; portanto, nesses casos, não é considerado como representativo do clone anormal. A baixa detecção de alteração cromossômica por cariótipo convencional em MM, em amostra de medula óssea, é bem reconhecida, pois na imensa maioria dos casos (50\%-70\%) o cariótipo revela metáfases normais pertencentes aos demais elementos mielóides da medula, em razão do fato de os plasmócitos terem um ciclo celular lento em relação às demais células não-malignas da medula ${ }^{(10,14)}$. Uma conseqüência dessa diferença na cinética celular é que, para a identificação de clone anormal, são necessárias análises em um grande número de células.

Entre os casos com resultado de cariótipo disponível neste estudo, 21\% apresentaram alterações, um percentual em conformidade com o descrito na literatura ${ }^{(4,14)}$. Entretanto, tem sido apontado que quando se observa alteração cromossômica em MM, pode-se inferir que se trata de célula com atividade proliferativa aumentada e, conseqüentemente, maior potencial de agressividade. De fato, todos os casos com cariótipo anormal, nesta casuística apresentavam $\beta 2 \mathrm{MG}$ e creatinina elevadas. A freqüência de alterações cromossômicas se correlaciona com o estágio da doença, sendo, portanto, mais freqüente em pacientes com extensa massa tumoral e, como conseqüência, com intensa atividade proliferativa( ${ }^{(6)}$.

$\mathrm{O}$ uso de FISH produziu grande efeito na demonstração de alterações cromossômicas nos pacientes deste estudo, pois permitiu a ampliação da detecção de anomalias. Com efeito, aneuploidias (alterações numéricas como monossomia ou trissomia) são detectadas em $80 \%-90 \%$ dos casos de MM, geralmente por estudo citogenético molecular $(\mathrm{FISH})^{(2)}$, embora, evolutivamente, também pelo clássico. Porém, nesta casuística, a elevada taxa observada pode ser devida ao estágio agressivo da doença diagnosticado na maioria dos pacientes.

Uma importante característica da instabilidade genômica é a falha da célula em manter o número correto de cromossomos. O processo que modifica o número de cromossomos é diferente daquele que causa mutações ou translocações. Apenas 10\% dos casos hiperdiplóides têm rearranjos cromossômicos, enquanto isso ocorre em $70 \%$ dos demais casos. Esse achado sugere que o MM pode seguir duas vias distintas, baseando-se na instabilidade genômica: aqueles com translocação e os demais com hiperploidia. A importância prognóstica e terapêutica desses dois grupos ainda é objeto de investigação, mas é muito provável que eles se comportem de formas diferentes ${ }^{(6,12)}$

As anormalidades cromossômicas numéricas permitem separar os pacientes em dois subgrupos biologicamente distintos: aqueles com hiperdiploidia versus os demais nãohiperdiplóides (onde estão inseridos os casos hipodiplóides, pseudodiplóides e hipotetraplóides) ${ }^{(3,12)}$. Há também notável associação entre não-hiperdiplóide e monossomia do cromossomo $13^{(3)}$. Fato assinalado em $64 \%$ (5/8, casos 4 , $5,8,11$ e 20) dos casos aqui estudados.

As anomalias do cromossomo 13 constituem um elemento biológico peculiar relacionado à sobrevida mais curta e a pior taxa de resposta ao tratamento ${ }^{(15)}$. O achado de $94 \%$ de pacientes em estádio de Durie e Salmon, avançado ou com marcadores desfavoráveis, tanto pode refletir uma questão de diagnóstico tardio numa parcela da população economicamente menos favorecida, a qual é atendida em instituições públicas no país (e para tanto esses dados devem ser confirmados), como pode apontar para a necessidade de medidas para possibilitar um diagnóstico mais precoce. Assim, esses pacientes poderão em breve se beneficiar do recente desenvolvimento nas áreas genômica e proteômica em MM, que permitiram avanços no entendimento da patogênese da doença e, conseqüentemente, identificaram novos alvos terapêuticos, viabilizando uma sustentação científica para a combinação de terapias que visem maior toxicidade para a célula tumoral e bloqueio da resistência à droga. Resta esclarecer, no entanto, se, nesses casos, o estágio avançado e/ou a doença agressiva implicam em resistência a tratamento.

Em relação ao lócus $\mathrm{IGH}$, que esteve envolvido em rearranjos em $25 \%$ dos casos estudados, esse pode refletir diferentes translocações, mutações ou recombinações en- 
volvendo a região variável do gene da imunoglobulina. Os parceiros podem ser os cromossomos 4, 6 e 16.

\section{Conclusão}

Encontrou-se alta incidência de alterações cromossômicas numéricas por FISH multicentromérico em MM em estágio avançado. Ao se agruparem os pacientes com hipodiploidia com aqueles com del13q, e compará-los com os demais casos, houve tendência a acúmulo de pacientes jovens no grupo com anomalias desfavoráveis $(p=0,06)$. Porém, o grupo hipo/del13q apresentou níveis de hemoglobina significativamente mais baixos $(\mathrm{Hb}<3,5$ $\mathrm{g} / \mathrm{dl}, p=0,03)$.

\section{Referências}

I. AVET-LOISEAU, H. et al. 14q32 translocations and monosomy 13 observed in monoclonal gammopathy of undetermined significance delineate a multistep process for the oncogenesis of multiple myeloma: Intergroupe Francophone du Myelome. Cancer Research, v. 59, p. 4546-50, 1999

2. CIGUDOSA, J.C. et al. Characterization of nonrandom chromosomal gains and losses in multiple myeloma by comparative genomic hybridization. Blood, v. 91, p. 300710, 1998.

3. DEBES-MARUN, C et al. Chromosome abnormalities clustering and its implications for pathogenesis and prognosis in myeloma. Leukemia, v. 17, p. 427-36, 2003.

4. DRACH, J. et al. Interphase fluorescence in situ hybridization identifies chromosomal abnormalities in plasma cells from patients with monoclonal gammopathy of undetermined significance. Blood, v. 86, p. 39I5-21, 1995.

5. DURIE, B.G.; SALMON, S.E. A clinical staging system for multiple myeloma: correlation of measured myeloma cell mass with presenting clinical features, response to treatment, and survival. Cancer, v. 36, p. 842-54, 1975.

6. FONSECA, R. et al. Clinical and biologic implications of of recurrent genomic aberrations in myeloma. Blood, v. I0I, n. II, p. 4569-75, 2003.

7. GREIPP, P.R. et al. International staging system for multiple myeloma. Clin Oncol, v. 23, n. I 5, p. 3412-20, 2005
8. HALLEK, M.; BERGSAGEL, P.L.; ANDERSON, K.C. Multiple myeloma: increasing evidence for a multipstep transformation process. Blood, v. 91 , n. I, p. 3-21, 1998.

9. HAROUSSEAU, J.-L. Corrected to Avet-Loiseau, Herve. J Clin Oncol, v. 23, n. 25, p. 628I, 2005. Erratum in: International staging system for multiple myeloma.

I0. LAI, J.L. et al. Improved cytogenetics in multiple myeloma: a study of I5 I patients including 117 patients at diagnosis. Blood, v. 85, p. 2490-7, 1995.

I I. MUNSHI, N.C. Recent advances in the management of multiple myeloma. Seminars in Hematology, v. 4I, n. 2, p. 21-6, 2004.

12. SMADJA, N.V. et al. Hypodiploidy is a major prognostic factor in multiple myeloma. Blood, v. 98, n. 7, p. 2229-38, 2001.

I 3. SMADJA, N.V. et al. Chromosomal analysis in multiple myeloma: cytogenetic evidence of two different diseases. Leukemia, v. 12, p. 960-9, 1998.

14. ZANDECKI, M.; LAI, J.L.; FACON, T. Multiple myeloma: almost all patients are cytogenetically abnormal. Brithish Journal of Haematology, v. 94, p. 217-27, 1996.

15. ZOJER, N. et al. Deletion of | $3 q \mid 4$ remains an independent adverse prognostic variable in multiple myeloma despite its frequent detection by interphase fluorescence in situ hybridization. Blood, v. 95, p. 1925-30, 2000. 